\title{
Recent progresses in ab-initio studies of low-energy few-nucleon reactions of astrophysical interest
}

Received: date / Accepted: date

\begin{abstract}
We review the most recent theoretical studies of nuclear reactions of astrophysical interest involving few-nucleon systems. In particular, we focus on the radiative capture of protons by deuterons in the energy range of interest for Big Bang Nucleosynthesis. Related to this, we will discuss also the most recent calculation of tritium $\beta$-decay. Two frameworks will be considered, the conventional and the chiral effective field theory approach.
\end{abstract}

Keywords Radiative proton-deuteron capture - Tritium $\beta$-decay

\section{Introduction}

The traditional picture of a nucleus views it as consisting of nucleons which interact among themselves and with external electroweak probes. This picture can be extensively tested by comparing theoretical and experimental results for many observables as, for instance, form factors, magnetic moments, and capture cross sections. Capture cross sections, then, are of particular interest, since in the low-energy regime they are difficult to be measured and theoretical predictions obtained in a well-tested framework become crucial inputs for astrophysics, in particular for stellar evolution models and for the theory of Big Bang Nucleosynthesis (BBN). Here we focus on the radiative proton capture on deuteron, $p+d \rightarrow^{3}$ $\mathrm{He}+\gamma(p d)$. An other significant example is the weak proton capture on proton, $p+p \rightarrow d+e^{+}+\nu_{e}$ $(p p)$. Although recent results are available also for this reaction (see Refs. $[1 ; 2 ; 3]$ ), due to lack of space, we will not discuss them in this contribution. The $p d$ reaction is of interest for the theory of BBN, since it is the main process through which deuterons are destroyed, and therefore it strongly affects the primordial deuterium abundance. Very recently, in order to achieve agreement between the measured primordial deuterium abundance and the BBN prediction, it has been questioned the accuracy of the only available experimental data in the BBN region. Therefore, an ab-initio calculation, as well as a more precise direct measurement, have become very urgent. The theoretical study has been performed in Ref. [4], while the experimental measurement is currently underway at the Gran Sasso Laboratories (Italy) by the LUNA Collaboration.

Related to the reactions of astrophysical interest, other processes are significant, since they can be used either to fix the unknown parameters of the theory, or to test the whole theoretical framework by comparing theory with available experimental results. Examples of these processes are tritium $\beta$ decay and muon captures on deuteron and ${ }^{3} \mathrm{He}$, as well as charge and magnetic form factors and static

L.E. Marcucci

Department of Physics "E. Fermi", University of Pisa

and INFN-Pisa

Largo B. Pontecorvo, 3

56127 Pisa (Italy)

E-mail: laura.elisa.marcucci@unipi.it 
properties of light nuclei. Of all these observables, we will consider here the Gamow-Teller matrix element of tritium $\beta$-decay (GT).

The present contribution, which is a review of the work of Ref. [4] for the $p d$ reaction, and of Ref. [5] for the tritium $\beta$-decay, is organized as follows: in Secs. 2 and 3 we briefly outline the theoretical frameworks used to perform these studies, i.e. the conventional phenomenological approach (ConvAp) and the chiral effective field theory approach $(\chi \mathrm{EFT})$, respectively. In Sec. 4 we review the results for the $p d$ reaction, and their implications for BBN, and for the tritium $\beta$-decay in $\chi \mathrm{EFT}$. In Sec. 5 , we summarize and give an outlook for this research activity.

\section{The conventional approach}

The conventional approach is based on realistic models for the nuclear interactions and currents. The nuclear interaction includes both two- $(N N)$ and three-nucleon $(3 N)$ potentials. These are constructed to reproduce the $A=2$ large body of experimental data with a $\chi^{2} /$ datum $\sim 1$, and the $A=3$ binding energies. In the present study, we use the Argonne $v_{18}$ (AV18) model [6] for the $N N$ interaction, and the Urbana IX (UIX) model [7] for the $3 N$ interaction (TNI). These models are quite complex and in parallel to their development, it is necessary to have at hand an ab-initio technique to solve the $A=3$ bound and scattering problem. To this aim, we use the hyperspherical harmonics (HH) technique, a variational approach which adopts a basis of $\mathrm{HH}$ functions to expand the bound or the scattering wave functions (in this second case, for relative interparticle distances small compared with the interaction range). A review of the method is given in Ref. [8], and references therein. Here, we would like to remark that the $\mathrm{HH}$ method is the only available one able to calculate the nuclear wave function for the $p d$ scattering state at low relative energies, as the ones of interest for BBN, including the Coulomb interaction between the charged initial particles. The $A=3$ bound and scattering properties, as the triton and ${ }^{3} \mathrm{He}$ binding energies, the $n d$ scattering length and elastic scattering observables as cross sections and analyzing powers are in general nicely reproduced by the AV18/UIX potential model, used in conjunction with the HH technique. Some discrepancies appear only for few delicate polarization observables (see for instance Ref. [9]).

The interaction between the considered nuclear systems and the external electroweak probe implies the construction of 6 operators, the electromagnetic current and charge operators, and the weak axial and vector current and charge operators. The number of operators reduces to 4 , since the conservedvector-current $(\mathrm{CVC})$ constraint relates the weak vector operators with the electromagnetic counterparts, just by performing a rotation in the isospin space (see for instance Ref. [10] and references therein). Here we only consider the nuclear electromagnetic and weak axial current operators. A review of all different contributions can be found in Refs. [11; 12; 13].

The nuclear electromagnetic current operator is constructed so that to satisfy the current conservation relation (CRC) exactly with the adopted Hamiltonian. The one-body term, the so-called impulse approximation (IA), obtained performing a $1 / m$ expansion ( $m$ is the nucleon mass) of the single-nucleon covariant current, satisfies CRC with the kinetic energy operator. The two-body electromagnetic current consists of two contributions, the so-called model-independent (MI) and model-dependent (MD) terms. The MI contributions have been constructed in Ref. [14], where particular care has been put to verify CRC with the AV18 potential. In particular, the two-body currents related to the momentumspace components of the two-body potential (spin-orbit $L \cdot S, L^{2}$ and $(L \cdot S)^{2}$ operators) are difficult to treat in the meson-exchange scheme and a new procedure based on minimal substitution has been devised. In addition, in order to satisfy CRC with the "full" Hamiltonian operator, which also include a three-body interaction term (here the UIX model), three-body electromagnetic currents need to be considered. They have been constructed in Ref. [14] both in the meson-exchange and minimal substitution scheme. The three-body currents, although they have found to give small contribution, are essential to describe correctly those observables sensitive to current conservation, as the $T_{20}$ and $T_{21}$ polarization observables in radiative capture of polarized deuterons on protons. The MD terms of the electromagnetic current are due to the $\rho \pi \gamma$ and $\omega \pi \gamma$ transitions and to the current associated with the excitation of one intermediate $\Delta$ resonance. These terms are transverse, and therefore they do not affect CRC. In the calculation for the $p d$ radiative capture of Ref. [4], which will be reviewed below, a new one-body term is included in the nuclear electromagnetic current, which is a relativistic correction of the order $1 / \mathrm{m}^{3}$ to the leading $1 / \mathrm{m}$ term. It was first derived in Refs. $[15 ; 16]$ in the context of 
chiral effective field theory, and it was found in Ref. [17] that it reduces the $n d$ radiative capture total cross section at thermal energies of about $4-5 \%$, bringing the theoretical prediction in a much better agreement with the experimental datum (within $4 \%$ ).

The weak axial current operator retains as well one- and two-body contributions. On the contrary to the electromagnetic case, there is no CRC for the axial case. Therefore, the two-body contributions should be seen as completely model-dependent. They can be divided in two classes: the operators of the first class are derived from $\pi$ - and $\rho$-meson exchanges and the $\rho \pi$-transition mechanism. These mesonic operators give rather small contributions. The operators in the second class are those that give the largest two-body contributions, and are due to $\Delta$-isobar excitation $[12 ; 13]$. In particular, in the dominant $N$-to- $\Delta$-transition axial current, the $N$-to- $\Delta$ axial coupling constant $\left(g_{A}^{*}\right)$ is retained as a parameter and is determined by fitting the experimental GT (GT ${ }^{\mathrm{EXP}}$ ). It is important to note that the value of $g_{A}^{*}$ depends on how the $\Delta$-isobar degrees of freedom are treated. In the present studies we derived the two-body $\Delta$-operator in static $\Delta$ approximation, using first-order perturbation theory. This approach is considerably simpler than that adopted in Ref. [13], where the $\Delta$ degrees of freedom were treated non-perturbatively, within the so-called transition-correlation operator approach, by retaining them explicitly in the nuclear wave functions. The results for $g_{A}^{*}$ obtained within the two schemes differ by more than a factor of 2 [13], but the results for the observables calculated consistently within the two different approaches are typically within $1 \%$ of each other.

\section{The chiral effective field theory approach}

Although the ConvAp has been proven to be very successful in describing the electroweak structure of light nuclei, it presents an "original sin", i.e., no connection with the underlying theory of strong interaction, quantum chromodynamics (QCD), is clearly visible. An alternative approach now available is the so-called chiral effective field theory $(\chi \mathrm{EFT})$ one. In a very schematic view, $\chi \mathrm{EFT}$ can be seen as a formulation of QCD in terms of effective degrees of freedom suitable for low-energy nuclear physics: pions and nucleons. The symmetries of QCD, in particular its (spontaneously broken) chiral symmetry, severely restrict the form of the interactions of nucleons and pions among themselves and with external electroweak fields, and make it possible to expand the Lagrangian describing these interactions in powers of $Q / \Lambda_{\chi}$, where $Q$ is the pion momentum and $\Lambda_{\chi}$ is the chiral-symmetry-breaking scale. It is usually set $\Lambda_{\chi} \sim 700 \mathrm{MeV}$, i.e. approximately the $\rho$-meson mass, or $\Lambda_{\chi} \sim 1 \mathrm{GeV}$, i.e. approximately the nucleon mass. In the studies presented here [5], we use $\Lambda_{\chi} \sim 1 \mathrm{GeV}$. As a consequence, classes of Lagrangians emerge, each of the order $\left(Q / \Lambda_{\chi}\right)^{n}$ and each involving a certain number of unknown coefficients, the so-called low-energy constants (LECs), which arise when the high-energy degrees of freedom are integrated out. While these LECs could in principle be determined by theory (for instance, in lattice QCD calculations), they are in practice constrained by fits to experimental data. The potentials and currents derived within this framework have power-law behavior for large momenta, and are regularized by introducing a momentum-cutoff function. It is expected that increasing the order of the chiral expansion $n$, the dependence on such cutoff $\Lambda$ will become weaker. Two aspects of the $\chi \mathrm{EFT}$ framework are very important: first of all, it is possible to derive in a very natural way nuclear electroweak currents consistently with the nuclear interaction, just by adding the external electroweak fields among the degrees of freedom of the theory. However, this has not yet been completely achieved, since the nuclear interactions and currents typically present different cutoff functions and are therefore not completely consistent. A complete consistency at this level is highly desirable, and work along this line is currently underway. Secondly, it is possible to set a hierarchy among the different contributions, both for the interactions and the currents, which allows to estimate the theoretical uncertainty arising from the truncation to a given order. In fact, lot of studies have been recently performed in order to quantify these uncertainties (see for instance Ref. [18]). Here, we apply the "naive" procedure of studying just the $\Lambda$-dependence of the observables. Also in $\chi \mathrm{EFT}$, two- and $3 N$ interactions, which in fact in this approach arise naturally, are used to describe the few-nucleon systems. In the present studies, we will use the $N N$ interaction derived by Entem and Machleidt in Ref. [19] (see also Ref. [20] and references therein) at next-to-next-to-next-to leading order (N3LO) and the TNI derived at next-to-next-to leading order (N2LO), in the local form of Ref. [21].

The idea of using $\chi \mathrm{EFT}$ to derive the nuclear electroweak transition operators was first implemented by Park et al [22] in the nineties for the electromagnetic current and charge operators and few 


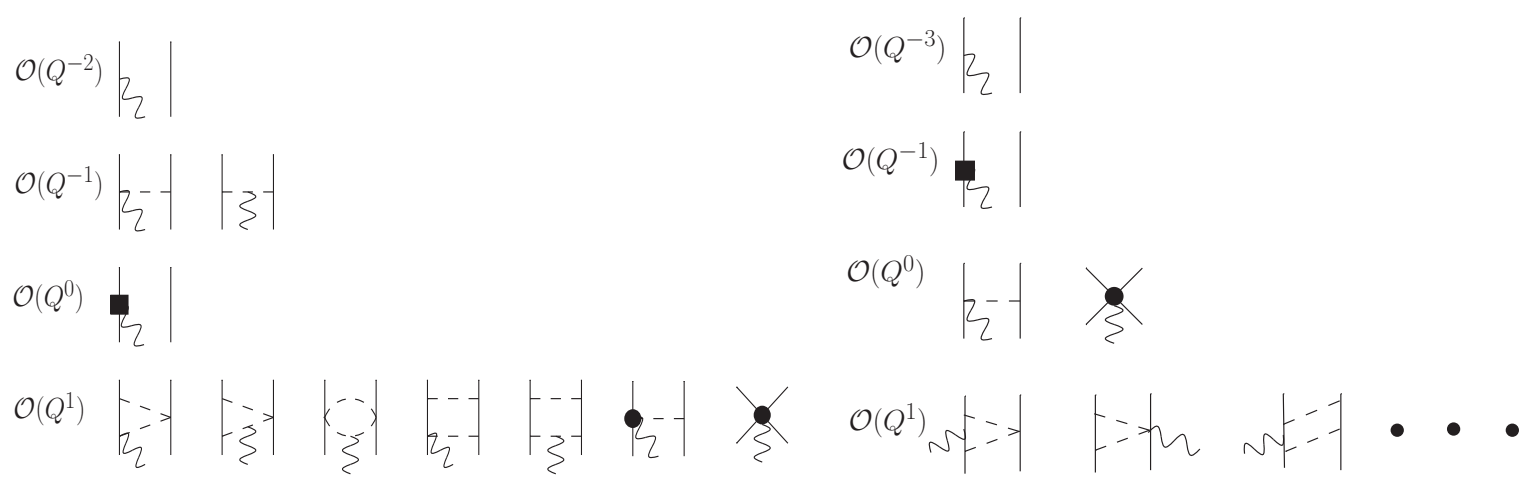

Fig. 1 On the left: diagrams illustrating one- and two-body $\chi$ EFT electromagnetic currents entering at LO $\left(Q^{-2}\right)$, NLO $\left(Q^{-1}\right)$, N2LO $\left(Q^{0}\right)$, and N3LO $\left(Q^{1}\right)$. On the right: diagrams illustrating one- and two-body $\chi$ EFT axial currents entering at LO $\left(Q^{-3}\right)$, NLO $\left(Q^{-1}\right)$, N2LO $\left(Q^{0}\right)$, and N3LO $\left(Q^{1}\right)$. Nucleons, pions, and electroweak probes are denoted by solid, dashed, and wavy lines, respectively. The solid square represents the relativistic corrections to the one-body current, while the solid circles represent the contact terms. Only the relevant topologies are indicated on the left, and only three of all possible N3LO contributions are shown on the right. The full listing of the N3LO diagrams for the axial current can be found in Ref. [28].

years later [23] for the weak axial current and charge operators. The so-called heavy-baryon chiral perturbation theory $(\mathrm{HB} \chi \mathrm{PT})$ approach was used, where the baryons are treated as heavy static sources, and the perturbative expansion is performed in terms of the involved momenta over the baryon mass. The developed currents have been the only one available in the $\chi \mathrm{EFT}$ framework for several years, and have been used in conjunction with the N3LO/N2LO $\chi$ EFT potentials to study weak processes which involve few-nucleon systems, as muon captures on light nuclei [24] and the proton weak capture on proton [1].

Few years ago, the problem of deriving the electromagnetic current and charge operators in $\chi \mathrm{EFT}$ has been revisited by Pastore et al [15; 25] and, in parallel, by Kölling et al [26; 27]. Pastore et al have used time-ordered perturbation theory (TOPT) to calculate the electromagnetic transition amplitudes, which allows for an easier treatment of the so-called reducible diagrams than the $\mathrm{HB} \chi \mathrm{PT}$ approach. On the other hand, Kölling et al have used the method of unitary transformation, the same one used to derive the chiral potentials mentioned above. We will focus here only on the work of Pastore et al, but we would like to remark that the results obtained by these two groups, although with different methods, are in good agreement with each other. Finally, very recently TOPT has been applied to derive the weak axial current and charge operators at N3LO [28], and we will review the results obtained with these new current for tritium $\beta$-decay in Sec. 4. The use of these new weak axial currents for other processes is currently underway.

As in the case of the ConvAp, also here we focus on the electromagnetic and weak axial currents. The contributions to the electromagnetic current at the different chiral orders are shown in the left panel of Fig. 1. The leading order (LO) contribution consists of the well known single-nucleon convection and magnetization currents, is of order $\mathcal{O}\left(Q^{-2}\right)$, and has the same expression as the IA term in the ConvAp. The N2LO contribution arises from the $(Q / m)^{2}$ relativistic corrections to the previous contribution, and is therefore of order $Q^{0}$. It is the same relativistic correction to the $1 / m$ expansion mentioned above within the ConvAp, which has been included since the work of Ref. [17] in radiative captures. The next-to-leading order (NLO) term involves seagull and in-flight long-range contributions associated with one-pion exchange (OPE). The N3LO currents, therefore at order $\mathcal{O}\left(Q^{1}\right)$, consist of one-loop twopion-exchange (TPE) terms, OPE terms induced by $\gamma \pi N$ interactions beyond LO, and contact terms generated by minimal substitution in the four-nucleon contact interactions involving two gradients of the nucleon fields, as well as by non-minimal couplings to the electromagnetic field. The former are linked to the $\chi$ EFT potential at order $\mathcal{O}\left(Q^{2}\right)$ via current conservation, and therefore they involve the same LECs entering the $\chi \mathrm{EFT} N N$ interaction. These are taken from fits to the $N N$ scattering data. On the other hand, the LECs entering the non-minimal contact currents as well as those entering the N3LO OPE contribution need to be fixed to electromagnetic observables. The adopted fitting procedure is extensively discussed in Ref. [29]. Here we only summarize the main features: (i) the LECs multiplying isoscalar operators are fixed so as to reproduce the deuteron magnetic moment and 
the isoscalar combination of the $A=3$ magnetic moments $\left(\mu_{S}\right)$. (ii) In order to achieve "natural" values for the LECs multiplying isovector operators and not to spoil chiral convergence, two LECs have been fixed by saturating the $\Delta$-resonance (a common strategy adopted in the literature), and the last LEC has been fixed by fitting either the cross section for neutron-proton radiative capture at thermal energies, $\sigma_{n p}$, or the isovector combination of the $A=3$ magnetic moments, $\mu_{V}$. These two sets of LECs, called SET II and SET III, respectively, are explicitly given in Ref. [29], where it has been shown that the experimental value for $\sigma_{n p}\left(\mu_{V}\right)$ is reproduced within few percent when the LECs of SET III (SET II) are used. (iii) There are significant differences between the present model for the electromagnetic current and that of Park et al at N3LO, both for the box diagrams and the contact terms. In particular, the contact terms of Park et al are much simpler than those presented above and can be written as sum of two terms, one isoscalar and one isovector, with two different LECs in front. These LECs have been fitted in Refs. $[24 ; 1]$ to reproduce the isoscalar and isovector combinations of the $A=3$ magnetic moments. For a more detailed discussion of this point, see Ref. [25]. Finally, we note that the power-law behavior at large momenta of the $\chi$ EFT operators has been regularized via the introduction of a cutoff function of the form $\exp \left(-Q^{4} / \Lambda^{4}\right)$, where $\Lambda$ assumes the same values used for the $N N$ potential and TNI.

The weak axial current operators are diagrammatically represented in the right panel of Fig. 1. The LO contribution consists of the well known single-nucleon axial current, and is of order $\mathcal{O}\left(Q^{-3}\right)$. At order $\mathcal{O}\left(Q^{-2}\right)$ it turns out that there are no contributions, and therefore the NLO contribution is of order $\mathcal{O}\left(Q^{1}\right)$, and arises from the $(Q / m)^{2}$ relativistic corrections to the LO contribution. The N2LO currents consist of the OPE term and a contact term with LEC typically denoted as $d_{R}$. The N3LO contributions are due to loop and TPE terms, and they have been calculated for the first time in Ref. [5]. They are not all represented in Fig. 1. The diagrammatic representation and a full discussion of these contributions can be found in Refs. $[5 ; 28]$. Here we only note that the LEC $d_{R}$ can be related to the LEC $c_{D}$ entering one of the two contact terms present in the TNI at N2LO, via the relation [30] $d_{R}=\frac{m}{\Lambda_{\chi} g_{A}} c_{D}+\frac{1}{3} m\left(c_{3}+2 c_{4}\right)+\frac{1}{6}$, where $g_{A}$ is the single-nucleon axial coupling constant, $c_{3}$ and $c_{4}$ are LECs of the $\pi N$ Lagrangian, already part of the chiral $N N$ potential at NLO. Therefore, it has become common practice since the work of Ref. [31] to fit $c_{D}$ (and $c_{E}$ - the other LEC entering the N2LO TNI) to the triton binding energy and the GT ${ }^{\mathrm{EXP}}$. The values have been obtained in this way until very recently using the axial current just up to N2LO, since the N3LO contributions were not yet available. The resulting $c_{D}$ and $c_{E}$ values for different cutoffs $\Lambda=450,500$ and $600 \mathrm{MeV}$ can be found in Refs. $[24 ; 32 ; 33]$ and they have been used in Refs. $[24 ; 1 ; 34 ; 35 ; 32 ; 33]$ to study muon capture on deuteron and ${ }^{3} \mathrm{He}$, the $p p$ capture, the $A=3$ and 4 elastic scattering observables, and the nuclear matter equation of state in many-body perturbation theory, respectively. The first calculation of $c_{D}$ and $c_{E}$ including N3LO axial term has been performed in Ref. [5], where it has been shown that the values for the LECs are significantly different depending whether the axial current is calculated up to N2LO or N3LO. This will be discussed in Subsection 4.2.

\section{Results}

In this section we present the results for the $p+d \rightarrow^{3} \mathrm{He}+\gamma(p d)$ radiative capture reaction, and tritium $\beta$-decay. In the first case, the ConvAp is used, in order to have a gauge invariant theoretical framework, with electromagnetic currents exactly conserved. We will not be able to estimate the theoretical uncertainty, except for that one arising from the numerical solution of the $A=3$ scattering problem. In the calculation of tritium $\beta$-decay, we will review the first results obtained with the axial current operator expanded up to N3LO.

\subsection{The $p d$ reaction}

The astrophysical $S$-factor for the $p d$ radiative capture is crucial to determine the consistency of BBN theoretical prediction for deuterium abundance, the new Planck results, and the most recent experimental determination of such abundance. In the absence of an accurate experimental determination in the energy range of interest for BBN, 30-300 keV, the $p d$ reaction has been studied within the ConvAp, using the $\mathrm{HH}$ technique to calculate the initial scattering and final bound state wave functions, with 
the realistic AV18/UIX potential model, and the realistic model for the nuclear current operator described in Sec. 2. We remark that this model satisfies gauge invariance with the adopted Hamiltonian and retains the $1 / \mathrm{m}^{3}$ contribution in the one-body operator. The results of Ref. [4] can be summarized as follows: (i) the numerical uncertainty relative to the solution of the $A=3$ scattering problem in the $S$-factor is lower than $1 \%$. This should not be seen as a theoretical uncertainty of the calculation, as the numerical uncertainty is only one entry of the total uncertainty budget. However, it makes the theoretical prediction quite robust. (ii) The $1 / \mathrm{m}^{3}$ one-body contribution increases the $S$-factor by $1-3 \%$ over the whole energy range. (iii) The effect of this new ab-initio determination of the astrophysical $S$-factor on the primordial deuterium abundance has been studied using the numerical code PArthENoPE [36]. We have found then that the BBN predictions are in very good agreement with the Planck 2015 results and the experimental result of Ref. [37]. In particular, for the Planck 2015 value of the energy density in baryons, $\Omega_{b} h^{2}$, and for standard value for the effective neutrino number, $N_{\text {eff }}$, it has been found that the ratio between the deuterium and hydrogen abundances is ${ }^{2} \mathrm{H} / \mathrm{H}=(2.46 \pm 0.03 \pm 0.03) \times 10^{-5}$, where the two errors are due to nuclear rate and $\Omega_{b} h^{2}$ uncertainties, respectively. This result is in nice agreement with the experimental value of Ref. [37], $(2.53 \pm 0.04) \times 10^{-5}$. Of course, these results ought to be confirmed by direct measurement of the $p d S$-factor currently underway by the LUNA Collaboration. Finally, it should be mentioned that a theoretical ab-initio calculation of the $p d$ reaction within $\chi \mathrm{EFT}$ is currently underway. However, although the first studies for the electromagnetic structure of light nuclei $[29 ; 11]$ have shown to be very promising, at present, the consistency between the $\chi$ EFT nuclear potentials and electromagnetic currents necessary to satisfy exactly gauge invariance (as in the case of the ConvAp framework) has not been yet achieved, making the $\chi$ EFT results not completely reliable at the accuracy level necessary for BBN predictions.

\subsection{Tritium $\beta$-decay}

In Ref. [5] we evaluate GT in $\chi \mathrm{EFT}$, by using the model for the weak current operator described in Sec. 3. The trinucleon wave functions have been obtained with the HH method using the two- and TNI N3LO/N2LO. Within a "hybrid" approach, quite commonly used in the literature (see for instance the review of Ref. [38]), also the phenomenological combination AV18/UIX has been considered. We will not discuss the results obtained within this "hybrid" approach here.

The different contributions to the GT matrix element are given in Table 1 for two different values of the cutoff $\Lambda=500,600 \mathrm{MeV}$. They are labelled LO, NLO, N2LO, and N3LO depending on the chiral counting illustrated in Fig. 1. The OPE contributions, at N2LO and N3LO, have been separated from the rest, i.e. the contact term at N2LO, and the multi-pion-exchange and three-body terms at N3LO. Indeed, the most important three-body contributions present at N3LO are also considered [5]. The LEC appearing at N2LO has been set equal to unity in appropriate units, in order to see how large is the contact-term contribution. It will be fixed to reproduce the experimental value of GT. By inspection of the table, we can conclude that contributions due to loop corrections in the axial current, i.e. the multi-pion-exchange terms, are relatively large and comparable to the N2LO(OPE). Furthermore, all the N3LO corrections have opposite signs relative to the LO and N2LO(OPE). The $\mathrm{N} 2 \mathrm{LO}(\mathrm{OPE})$ contributions are in fact suppressed, since the contributions proportional to the LECs $c_{3}$ and $c_{4}$ interfere destructively and therefore depend strongly on the values of these LECs. Note that the LECs $c_{3}$ and $c_{4}$ are already present in the $N N$ interaction at N2LO and therefore are fixed once the $N N$ potential is chosen.

In order to fix the LEC entering the N2LO contact term, we imposed to reproduce the experimental GT matrix element, $\mathrm{GT}^{\mathrm{EXP}}=0.9511 \pm 0.0013$, as redetermined in Ref. [5] by updating the value for the single-nucleon axial coupling constant $g_{A}$ and the Fermi constant and functions. The procedure to determine this LEC, $c_{D}$, in conjunction with the LEC $c_{E}$ entering the TNI at N2LO is the same as that of Ref. [24], i.e. we require to reproduce simultaneously the $A=3$ binding energy and $\mathrm{GT}^{\mathrm{EXP}}$. The values for $c_{D}$ and $c_{E}$ are given in Table 2. By inspection of the table we can conclude that, not surprisingly, the values for the LECs are strongly dependent on the chiral order retained in the axial current operator. The origin of this strong dependence can be traced back to the strong suppression of the N2LO(OPE) contributions mentioned above. The values of $c_{D}$ and $c_{E}$ obtained retaining only the N2LO contributions in the axial current have been widely used in the literature, as mentioned above. Therefore, we believe that it has become necessary to verify whether the presented results need to 
Table 1 Contributions to the GT matrix element of tritium $\beta$-decay obtained with the N3LO/N2LO nuclear Hamiltonian and cutoffs $\Lambda=500 \mathrm{MeV}$ and $600 \mathrm{MeV}$ in the chiral potentials and weak axial current operators. The acronyms LO, NLO, N2LO, and N3LO refer, respectively, to the axial operators given in Fig. 1 at $\mathcal{O}\left(Q^{-3}\right)$, $\mathcal{O}\left(Q^{-1}\right), \mathcal{O}\left(Q^{0}\right)$, and $\mathcal{O}\left(Q^{1}\right)$. At different orders, we have isolated the one-pion-exchange (OPE), multi-pionexchange (MPE), contact-term (CT) and three-body (3B) contributions. Furthermore, the LEC in N2LO(CT) is set equal to unity in appropriate units, in order to see the significance of this contact contribution.

\begin{tabular}{ccc}
\hline$\Lambda$ & $500 \mathrm{MeV}$ & $600 \mathrm{MeV}$ \\
\hline $\mathrm{LO}$ & 0.9363 & 0.9322 \\
$\mathrm{NLO}$ & $-0.569 \times 10^{-2}$ & $-0.457 \times 10^{-2}$ \\
$\mathrm{~N} 2 \mathrm{LO}(\mathrm{OPE})$ & $0.825 \times 10^{-2}$ & $0.043 \times 10^{-2}$ \\
$\mathrm{~N} 2 \mathrm{LO}(\mathrm{CT})$ & $-0.586 \times 10^{-3}$ & $-0.717 \times 10^{-3}$ \\
$\mathrm{~N} 3 \mathrm{LO}(\mathrm{OPE})$ & $-0.697 \times 10^{-2}$ & $-0.867 \times 10^{-2}$ \\
$\mathrm{~N} 3 \mathrm{LO}(\mathrm{MPE})$ & $-0.430 \times 10^{-1}$ & $-0.532 \times 10^{-1}$ \\
$\mathrm{~N} 3 \mathrm{LO}(3 \mathrm{~B})$ & $-0.143 \times 10^{-2}$ & $-0.153 \times 10^{-2}$ \\
\hline
\end{tabular}

Table 2 Values for the LECs $c_{D}$ and $c_{E}$ entering the axial current operator and the TNI, as obtained by fitting the $A=3$ binding energy and $\mathrm{GT}^{\mathrm{EXP}}$ (its central value), using the N3LO/N2LO potential models with cutoffs $\Lambda=500 \mathrm{MeV}$ and $600 \mathrm{MeV}$. The results labelled N2LO and N3LO are obtained retaining in the nuclear axial current up to $\mathcal{O}\left(Q^{0}\right)$ and $\mathcal{O}\left(Q^{1}\right)$ contributions, respectively. In addition, we show in the last column the value in $\mathrm{fm}$ for the $n d$ doublet scattering length.

\begin{tabular}{ccccc}
\hline$\Lambda[\mathrm{MeV}]$ & & $c_{D}$ & $c_{E}$ & $a_{n d}[\mathrm{fm}]$ \\
\hline 500 & N2LO & -0.353 & -0.305 & 0.665 \\
& N3LO & -1.847 & -0.548 & 0.654 \\
600 & N2LO & 0.443 & 1.224 & 0.699 \\
& N3LO & 2.030 & 1.553 & 0.688 \\
\hline
\end{tabular}

be revisited or do not change significantly. Studies along this line, in particular for the muon capture reactions, are currently underway.

In Table 2, we show also the results for the $n d$ doublet scattering length $\left(a_{n d}\right)$ calculated with the given combination of $c_{D}$ and $c_{E}$ in the nuclear Hamiltonian N3LO/N2LO. The experimental value for $a_{n d}$ is $0.645 \pm 0.010 \mathrm{fm}$. To be noticed that in the present calculation, we have ignored higher order electromagnetic interaction terms, such as those associated with the nucleons' magnetic moments. These terms are known to reduce the $a_{n d}$ value of about $3 \%$ [8], when the AV18/UIX Hamiltonian model is used. The results of Table 2 seem to indicate that the three $A=3$ observables $(A=3$ binding energies, $\mathrm{GT}^{\mathrm{EXP}}$, and $a_{n d}$ ) are simultaneously reproduced, at least for $\Lambda=500 \mathrm{MeV}$, when the nuclear axial current retains corrections up to N3LO.

\section{Conclusions and outlook}

We have presented the most recent studies of low-energy reactions which involve light nuclei within the conventional approach and the most recent $\chi$ EFT framework. In particular, we have shown that the first steps have been done in order to study weak processes as muon capture on light nuclei and the weak proton captures by proton or ${ }^{3} \mathrm{He}$ using a model for the weak axial current operator which retains contributions up to $\mathcal{O}\left(Q^{1}\right)$. Work along this line are currently underway.

\section{References}

1. Marcucci L E, Schiavilla R, Viviani M (2013) Proton-proton weak capture in chiral effective field theory. Phys. Rev. Lett. 110: 192503

2. Tognelli E, Degl'Innocenti S, Marcucci L E, Prada Moroni P G (2015) Astrophysical implications of the proton-proton cross section updates. Phys. Lett. B 742: 189-194

3. Acharya B, Carlsson B D, Ekström A, Forssèn C, Platter L (2016) Uncertainty quantification for protonproton fusion in chiral effective field theory. Phys. Lett. B 760: 584-589

4. Marcucci L E, Mangano G, Kievsky A, Viviani M (2016) Implication of the proton-deuteron radiative capture for Big Bang Nucleosynthesis. Phys. Rev. Lett. 116: 102501; Erratum Phys. Rev. Lett. 117: 049901 
5. Baroni A, Girlanda L, Kievsky A, Marcucci LE, Shiavilla R, Viviani M (2016). Tritium $\beta$-decay in chiral effective field theory. Phys. Rev. C 94: 024003

6. Wiringa R B, Stoks V G J, Schiavilla R (1995) Accurate nucleon-nucleon potential with chargeindependence breaking. Phys. Rev. C 51: 38-51

7. Pudliner B S, Pandharipande V R, Carlson J, Pieper S C, Wiringa R B (1997) Quantum Monte Carlo calculations of nuclei with $A \leq 7$. Phys. Rev. C 56: 1720-1750

8. Kievsky A, Rosati S, Viviani M, Marcucci L E, Girlanda L (2008) A High-precision variational approach to three- and four-nucleon bound and zero-energy scattering states. J. Phys. G 35: 063101

9. Marcucci L E, Kievsky A, Girlanda L, Rosati S, Viviani M (2009) N - d Elastic Scattering Using the Hyperspherical Harmonics Approach with Realistic Local and Non-Local Interactions. Phys. Rev. C 80: 034003

10. Marcucci L E et al (2011) Muon capture on deuteron and ${ }^{3}$ He. Phys. Rev. C 83: 014002

11. Marcucci L E et al (2016) Electromagnetic structure of few-nucleon ground states. J. Phys. G 43: 023002

12. Schiavilla R et al (1998) Weak capture of protons by protons. Phys. Rev. C 58: 1263-1277

13. Marcucci L E et al (2000) Weak proton capture on ${ }^{3}$ He. Phys. Rev. C 63: 015801

14. Marcucci L E, Viviani M, Schiavilla R, Kievsky A, Rosati S (2005) Electromagnetic structure of $A=2$ and 3 nuclei and the nuclear current operator. Phys. Rev. C 72: 014001

15. Pastore S, Girlanda L, Schiavilla R, Viviani M, Wiringa R B (2009) Electromagnetic Currents and Magnetic Moments in $\chi$ EFT. Phys. Rev. C 80: 034004

16. Song Y-H, Lazauskas R, Park T-S (2009) Up to N $\mathrm{N}^{3} \mathrm{LO}$ heavy-baryon chiral perturbation theory calculation for the $M 1$ properties of three-nucleon systems. Phys. Rev. C 79: 064002

17. Girlanda L et al (2010) Thermal Neutron Captures on $d$ and ${ }^{3}$ He. Phys. Rev. Lett. 105: 232502

18. Carlsson B D et al (2016) Uncertainty analysis and order-by-order optimization of chiral nuclear interactions. Phys. Rev. X 6: 011019

19. Entem D R and Machleidt R (2003) Accurate charge dependent nucleon nucleon potential at fourth order of chiral perturbation theory. Phys. Rev. C 68: 041001

20. Machleidt R and Entem D R (2011) Chiral effective field theory and nuclear forces. Phys. Rep. 503:1-pp

21. Navràtil P (2007) Local three-nucleon interaction from chiral effective field theory. Few-Body Syst. 41: 117-140

22. Park T S, Min D P, Rho M (1996) Chiral Lagrangian approach to exchange vector currents in nuclei. Nucl. Phys. A 596: 515-552

23. Park T S et al (2003) Parameter-free effective field theory calculation for the solar proton-fusion and hep processes. Phys. Rev. C 67: 055206

24. Marcucci L E, Kievsky A, Rosati S, Schiavilla R, Viviani M (2012) Chiral Effective Field Theory Predictions for Muon Capture on Deuteron and ${ }^{3}$ He. Phys. Rev. Lett. 108: 052502

25. Pastore S, Girlanda L, Schiavilla R, Viviani M (2011) The two-nucleon electromagnetic charge operator in chiral effective field theory ( $\chi$ EFT) up to one loop. Phys. Rev. C 84: 024001

26. Kölling S, Epelbaum E, Krebs H, Meissner U G (2009) Two-pion exchange electromagnetic current in chiral effective field theory using the method of unitary transformation. Phys. Rev. C 80: 045502

27. Kölling S, Epelbaum E, Krebs H, Meissner U G (2011) Two-nucleon electromagnetic current in chiral effective field theory: One-pion exchange and short-range contributions. Phys. Rev. C 84: 054008

28. Baroni A, Girlanda L, Pastore S, Schiavilla R, Viviani M (2016) Nuclear Axial Currents in Chiral Effective Field Theory. Phys. Rev. C 93: 015501; Erratum in Phys. Rev. C 93: 049902

29. Piarulli $\mathrm{M}$ et al (2013) Electromagnetic structure of $A=2$ and 3 nuclei in chiral effective field theory. Phys. Rev. C 87: 014006

30. Gårdestig A and Phillips D R (2006) How Low-Energy Weak Reactions Can Constrain Three-Nucleon Forces and the Neutron-Neutron Scattering Length. Phys. Rev. Lett. 96: 232301

31. Gazit D, Quaglioni S, Navratil P (2009) Three-Nucleon Low-Energy Constants from the Consistency of Interactions and Currents in Chiral Effective Field Theory. Phys. Rev. Lett. 103: 102502

32. Coraggio L et al (2014) Nuclear-matter equation of state with consistent two- and three-body perturbative chiral interactions. Phys. Rev. C 89: 044321

33. Sammarruca F et al (2015) Toward order-by-order calculations of the nuclear and neutron matter equations of state in chiral effective field theory. Phys. Rev. C 91: 054311

34. Viviani M, Girlanda L, Kievsky A, Marcucci L E (2013) Effect of three-nucleon interaction in $p-{ }^{3} \mathrm{He}$ elastic scattering. Few-Body Syst. 54: 885-890

35. Viviani M Girlanda L, Kievsky A, Marcucci L E (2013) Effect of Three-Nucleon Interaction in $p-{ }^{3} \mathrm{He}$ Elastic Scattering. Phys. Rev. Lett. 111: 172302

36. Pisanti $\mathrm{O}$ et al (2008) PArthENoPE: Public algorithm evaluating the nucleosynthesis of primordial elements. Comput. Phys. Comm. 178: 956-971

37. Cooke R, Pettini M, Jorgenson R A, Murphy M T, Steidel C C (2014) Precision measures of the primordial abundance of deuterium. Astrophys. J. 781: 31

38. Bacca S and Pastore S (2016) Electromagnetic reactions on light nuclei. J. Phys. G 41: 123002 